\title{
Sepsis, CTCAE
}

National Cancer Institute

\section{Source}

National Cancer Institute. Sepsis, CT CAE. NCI Thesaurus. Code C143827.

A disorder characterized by the presence of pathogenic microorganisms in the blood stream that cause a rapidly progressing systemic reaction that may lead to shock. 